\title{
HIV-specific T-cell responses reflect substantive in vivo interactions with infected cells despite long-term therapy
}

\section{Authors and affiliations:}

Eva M. Stevenson ${ }^{1 \#}$, Adam R. Ward ${ }^{1,2,3 \#}$, Ronald Truong ${ }^{2}$, Allison S. Thomas ${ }^{4}$, Szu-Han Huang ${ }^{1,2}$, Thomas R. Dilling ${ }^{1}$, Sandra Terry ${ }^{1}$, John K. Bui ${ }^{1}$, Talia M. Mota ${ }^{1}$, Ali Danesh ${ }^{1}$, Guinevere Q. Lee ${ }^{1}$, Andrea Gramatica ${ }^{1}$, Pragya Khadka ${ }^{1}$, Winiffer D. Conce Alberto ${ }^{1}$, Rajesh T. Gandhi ${ }^{5}$, Deborah K. McMahon $^{6}$, Christina M. Lalama ${ }^{7}$, Ronald J. Bosch ${ }^{7}$, Bernard Macatangay ${ }^{6}$, Joshua C. Cyktor ${ }^{6}$, Joseph J. Eron ${ }^{8}$, John W. Mellors ${ }^{6}$, R. Brad Jones ${ }^{1,2 *}$, for the ACTG A5321 Team

${ }^{1}$ Division of Infectious Diseases, Weill Cornell Medicine, New York, NY USA

${ }^{2}$ Department of Microbiology, Immunology, and Tropical Medicine, George Washington University, Washington, DC USA

${ }^{3} \mathrm{PhD}$ Program in Epidemiology, George Washington University, Washington, DC USA

${ }^{4}$ Department of Microbiology, Boston University School of Medicine, Boston, MA USA

${ }^{5}$ Division of Infectious Diseases, Massachusetts General Hospital, Boston, MA USA

${ }^{6}$ Department of Medicine, University of Pittsburgh School of Medicine, Pittsburgh, PA USA

${ }^{7}$ Center for Biostatistics in AIDS Research, Harvard T.H. Chan School of Public Health, Boston, MA USA

${ }^{8}$ Department of Medicine, University of North Carolina at Chapel Hill, Chapel Hill, NC USA

\#These authors contributed equally

${ }^{*}$ Corresponding author:

27 R. Brad Jones

28 rbjones@med.cornell.edu

29413 E $69^{\text {th }}$ St, $5^{\text {th }}$ floor

30 New York, NY 10021 


\section{Abstract}

43 Antiretroviral therapies (ART) durably suppress HIV replication to undetectable levels - however,

44 infection persists in the form of long-lived reservoirs of infected cells with integrated proviruses,

45 that re-seed systemic replication if ART is interrupted. A central tenet of our current understanding

46 of this persistence is that infected cells are shielded from immune recognition and elimination

47 through a lack of antigen expression from proviruses. Efforts to cure HIV infection have therefore

48 focused on reactivating latent proviruses to enable immune-mediated clearance, but these have

49 yet to succeed in driving reductions in viral reservoirs. Here, we revisited the question of whether

50 HIV reservoirs are predominately immunologically silent from a new angle, by querying the

51 dynamics of HIV-specific T-cell responses over long-term ART for evidence of ongoing

52 recognition of HIV-infected cells. We show that T-cell responses to autologous reservoir viruses

53 persist over years, and that the maintenance of HIV-Nef-specific responses was uniquely

54 associated with residual frequencies of infected cells. These responses disproportionately

55 exhibited a cytotoxic, effector functional profile, indicative of recent in vivo recognition of HIV-

56 infected cells. These results indicate substantial visibility of the HIV reservoir to T-cells on stable

57 ART, presenting both opportunities and challenges for the development of therapeutic

58 approaches to curing HIV infection. 


\section{Introduction}

The needs for both a vaccine and a cure for HIV are underscored by the ongoing impact

70 of this global pandemic, which continues to cause close to 800,000 deaths annually (1).

71 Antiretroviral therapy (ART) is capable of durably suppressing HIV replication, and halting disease

72 progression for those able to access and adhere to these regimens. Infection persists, however,

73 in reservoirs of $\mathrm{CD}^{+} \mathrm{T}$-cells, and potentially other cell types $(2,3)$ with integrated proviruses that

74 re-seed systemic replication if ART is interrupted $(2,4-9)$. These proviruses often exist in a latent

75 state, characterized by limited transcription and, presumably, a lack of antigen production. This

76 gives rise to one of the central tenets in the study of HIV persistence, which postulates that the

77 persistent reservoir (often called the 'latent reservoir') is not detected by the immune system in

78 individuals on long-term ART. It follows that engaging the immune system to reduce HIV

79 reservoirs depends upon latency reversal to re-expose the immune system to HIV antigen - the

80 so-called "kick and kill" (or "shock and kill") strategy (10).

While latency undoubtedly diminishes immune recognition of viral reservoirs, several lines

82 of evidence cast doubt on whether this is absolute in vivo, which would implicate additional

83 contributors to viral persistence (11). Most notably, unspliced, and sometimes multiply spliced,

84 HIV transcripts are readily detectable in peripheral blood mononuclear cells (PBMCs) of

85 individuals on durable ART $(12,13)$. These observations have recently led some to propose

86 amendments to the "latent reservoir" model, by introducing the idea of a continuum ranging from

87 "deep latency" (no RNA produced) through to an "active reservoir" (14, 15). A key unresolved

88 question, however, is whether these transcripts result in HIV-protein production, and thus enable

89 immune recognition. Multiple factors limit the degree to which this can be inferred from direct

90 measures of in vivo viral expression, including sampling difficulties - given that expression may

91 be anatomically or temporally restricted - and the lack of equivalency between readily measurable 
92 features (ex. viral RNA) with bonafide antigen presentation (16, 17). We therefore hypothesized

93 that some level of antigen recognition by HIV-specific T-cells may occur in vivo in ART-

94 suppressed individuals with undetectable viremia. We predicted that this would be reflected in

95 relationships between the long-term dynamics of HIV-specific T-cell responses and measures of

96 virologic persistence, including frequencies of infected cells.

Although the T-cell response to HIV infection has been generally well characterized, and

98 is known to decay rapidly in the months following ARV initiation (18-20), there are a lack of well-

99 powered studies that have addressed the long-term dynamics of these responses in association

100 with virologic parameters. In a previous cross-sectional study, we observed a modest correlation

101 between the magnitudes of T-cell responses to the HIV-Nef protein and residual frequencies of

102 infected cells, providing some initial suggestion that these responses may be maintained by

103 antigen recognition. However, a recent longitudinal study reported that, while responses were

104 highly stable on durable ART, no correlations were observed between response magnitudes and

105 reservoir size as measured by quantitative viral outgrowth assays across 18 individuals (21). The

106 current study builds upon these earlier reports by uniquely assessing T-cell response dynamics

107 over almost 3 years in association with multiple measures of viral persistence, in a cohort of 49

108 individuals on well-documented sustained ART. We first confirm that, in this cohort, T-cell

109 responses to autologous reservoir viruses are well represented by a scalable IFN- $\gamma$ enzyme-linked

110 immunospot (ELISPOT) assay, and show that these responses persist over years. Strikingly, the

111 persistence of T-cell responses to the HIV-Nef protein (slopes of change) over 144 weeks were

112 strongly and uniquely associated with the frequencies of infected cells that persisted on ART (22,

113 23), and these responses disproportionately exhibited a cytotoxic effector functional profile,

114 indicative of recent in vivo antigen recognition (24-28). These results conclusively reveal ongoing

115 interactions between the immune system and the HIV reservoir over years of ART, with 
116 implications both for understanding HIV persistence, and designing interventions aimed at curing

117 infection.

\section{Results}

$119 C D 8^{+}$T-cell Responses to Autologous Infected Cells

We approached the characterization of $\mathrm{CD}^{+} \mathrm{T}$-cell responses in our study with initial

121 concerns over putative limitations in conventional approaches to quantifying T-cell responses to

122 cells infected with autologous reservoir viruses. Namely, by utilizing synthetic peptides as

123 antigens, conventional approaches may: i) detect responses from T-cells that are unable to

124 recognize autologous reservoir viruses, as a result of sequence variation $(13,29)$; ii) not fully

125 capture the entirety of viral epitopes, which may also be expressed from cryptic reading frames,

126 or novel exon structures (23); and iii) skew representation of epitopes that are differentially

127 affected by processing in infected cells (30).

128 With the aim of more comprehensively quantifying the total ability of $\mathrm{CD}^{+} \mathrm{T}_{\text {-cells }}$ to 129 recognize infected cells, we developed a 'biosensor assay' whereby ex vivo CD8 ${ }^{+}$T-cells were 130 co-cultured with excess HIV-superinfected autologous $\mathrm{CD}^{+}{ }^{+}$T-cells. For each individual

131 (participant characteristics in Table S1), we prepared two sets of target cells infected with either:

132 i) the molecular clone of HIV, JRCSF, or ii) a cocktail of autologous reservoir viruses generated

133 by pooling the supernatants of quantitative viral outgrowth assays (Fig. 1A \& B). Flow cytometric

134 analysis detected CD8 ${ }^{+}$T-cells that responded to these infected cells by degranulating (CD107a)

135 and/or producing IFN- $\gamma$, in most individuals (Fig. 1C). In comparing these biosensor assay 136 responses (to autologous viruses) with the total IFN- $\gamma$ ELISPOT responses (summed across all

137 HIV gene products), we observed a strong correlation (Spearman $r=0.840, p=0.005$, Fig. 1D), in 138 spite of the above-noted putative limitations with using synthetic peptides. Thus, these data from 139 our 'biosensor assay' serve to not only directly demonstrate that CD8 ${ }^{+}$T-cell responses capable 
140 of recognizing cells infected with autologous reservoir viruses remain present in individuals on

141 long-term ART, but also to show that such responses are reasonably well represented by

142 ELISPOT results, when summed across all HIV gene products.

143 Magnitudes of T-cell Responses on Long-Term ART

With the above validation in place, we leveraged the scalability of the ELISPOT assay to

145 comprehensively examine T-cell response dynamics in a larger cohort. These assays were 146 performed using overlapping 15-mer peptides spanning: i) HIV-Gag, ii) HIV-Env, iii) HIV-Pol, iv) 147 HIV-Nef/Tat/Rev, v) HIV-Tat, vi) HIV-Rev, vii) HIV-Nef, and viii) CMVpp65 (control), with samples 148 from the ACTG A5321 HIV Reservoirs Cohort Study, which consists of participants who initiated 149 ART during chronic HIV infection and had subsequent well-documented, sustained virologic 150 suppression (undetectable by clinical assay prior to and throughout the study period) (31) (Fig. 2

151 and Table 1). We previously assessed HIV-specific T-cell responses in A5321 at study entry, a 152 median of 7 (range 4-15) years after ART initiation (32). Here, we extended these results with 153 batched analysis of samples from 24 and 168 weeks after study entry. IFN- $\gamma$-producing HIV154 specific T-cell responses were readily detected against Gag, Pol, and Nef, with median values at 15524 weeks: $103,78.5$, and 78.5 SFU $/ 10^{6}$ PBMCs, respectively; and at 168 weeks: $87.0,44.7$, and 156 43.3 SFU/10 6 PBMCs, respectively (Fig. 3A\&B and Table S2).

157 Between this 24 to 168 week period, time-averaged responses against Gag were the 158 highest, and significantly greater than responses to Env, Nef, Tat, and Rev (all $p<0.05)($ Table 159 S3). Notably, T-cell responses directed against Tat and Rev were the lowest in magnitude, and 160 negligible at both timepoints (Fig. 3B and Tables S2 \& S3), Env-specific responses were also 161 low, with median values of 22.9 and 10.3 SFU/10 $10^{6}$ PBMCs at 24 and 168 weeks, respectively 162 (Fig. 3B and Table S2). The long-term persistence of HIV-specific T-cell responses - primarily 
163 directed against Gag, Pol, and Nef - over years of ART thus provided initial support for these

164 HIV-specific T-cells continuing to interact with persisting infected cells.

To further characterize the long-term dynamics of HIV-specific T-cell responses in A5321

167 cohort participants on durable ART, we categorized participants' IFN- $\gamma$ ELISPOT responses from

168 the batched 24 to 168 weeks post-study entry data as either increasing, decreasing, or not

169 changing (defined as $\leq 15 \%$ change), and observed considerable heterogeneity (Fig. S1).

170 Notably, population-average responses to Nef, summed HIV, and CMV-pp65 did not decline

171 significantly over this 144 week time period, whereas responses to Gag, Env, and Pol all showed

172 significant declines over time (Fig. 4A and Table S4). However, all HIV-specific T-cell responses

173 demonstrated remarkable persistence, with the responses which showed a significant decline

174 only averaging between $0.35 \%$ to $0.62 \%$ loss per week in IFN- $\gamma$ ELISPOT assays (Table S4).

To determine whether ongoing antigen recognition by HIV-specific T-cells could be

176 maintaining IFN- $\gamma$-producing HIV-specific T-cell responses, we next examined associations

177 between the slopes of change of T-cell response magnitudes between 24 and 168 weeks post-

178 study entry (based on absolute changes on a linear scale) with on-ART virologic parameters,

179 including total cell-associated HIV DNA (CA-DNA), cell-associated HIV RNA (CA-RNA), and

180 plasma HIV RNA by integrase single copy assay (iSCA). The dynamics of responses to the most

181 immunogenic antigens, Gag and Nef (33), along with summed HIV responses, were significantly

182 associated with pre-ART viral loads (Fig. 4B and Table S5), despite participants having been on

183 ART for over a median of 7 years when responses were first measured. Strikingly, however, the

184 slopes of change in Nef-specific responses were unique in exhibiting highly significant direct

185 associations with any on-ART virologic parameter after controlling for potential confounding by

186 pre-ART plasma viral load and pre-ART CD4 ${ }^{+}$T-cell count, specifically on-ART CA-DNA ( $r=$ 
0.496, $p=0.003)$ and CA-RNA $(r=0.405, p=0.019)$ at study entry (Fig. 4B and Table S5).

188 These results indicate that both higher frequencies of persistent infected cells (CA-DNA) and

189 higher levels of viral transcription (CA-RNA) were associated with greater maintenance of Nef-

190 specific responses, consistent with ongoing stimulation by infected cells. Slopes of change in HIV-

191 specific T-cell responses were not associated with PD-1 levels on total CD4 ${ }^{+}$or CD8 ${ }^{+} \mathrm{T}_{\text {-cells (Fig. }}$

192 4B and Table S5), but generally correlated with each other (Table S6). Analyzing slopes of

193 change in $\log _{10}$-transformed T-cell response magnitudes, reflecting proportional changes in

194 responses rather than absolute changes, revealed significant associations between the dynamics

195 of Nef-specific, Nef/Tat/Rev-specific, and summed HIV-specific T-cell responses with on-ART CA-

196 DNA at study entry (all $p<0.05$ - Table S7), with proportional changes in HIV-specific responses

197 generally correlating with each other (Table S8). Thus, whether dynamics were measured on an

198 absolute or proportional change scale, Nef-specific response persistence was uniquely

199 associated with HIV-infected cell frequencies. These results suggest that Nef-specific T-cell

200 responses are preferentially maintained by ongoing interactions with HIV-infected cells, though

201 all responses are likely maintained to some extent by ongoing HIV antigen recognition given their

202 exceptional persistence.

203 Recent In Vivo Antigen Recognition by Nef-Specific T-cells

204 We next investigated whether the functional properties of HIV-specific CD8 ${ }^{+}$T-cells would

205 yield insights into their recent histories of in vivo antigen encounter. Data from human studies and

206 animal models have highlighted ex vivo granzyme B production as a distinguishing feature of

207 virus-specific effector $\mathrm{CD}^{+}{ }^{+}$T-cells which have recently encountered antigen in vivo - either

208 through infection or vaccination (24-28). While granzyme B production can be induced in memory

$209 \mathrm{CD}^{+}$T-cells, this requires more than 24 hours of in vitro stimulation, whereas IFN- $\gamma$ is produced

210 rapidly from both memory and effector $\operatorname{CD}^{+}$T-cells $(24,34,35)$. Thus, ex vivo ELISPOT

211 measurements of granzyme B have been established as an 'immune diagnostic' means of 
212 identifying effector responses to active infections $(34,36)$. To quantify the effector functionalities

213 of HIV-specific T-cells on long-term ART, we performed batched granzyme B ELISPOT assays

214 on week 24 and 168 samples (Fig. 5A). We focused on the Gag, Pol, and Nef peptide pools,

215 having observed these to be the most immunogenic by IFN- $\gamma$ ELISPOT. Overall, granzyme B-

216 producing HIV-specific responses were substantially lower in magnitude than IFN- $\gamma$ responses

217 (Fig. 5B, 5C and Table S2). The median magnitudes of granzyme B responses relative to each

218 other were: Nef>Pol>Gag (at both timepoints - Fig. 5B and Table S2), contrasting with IFN- $\gamma$ :

219 Gag>Pol =Nef (Fig. 3B and Table S2). As with IFN- $\gamma$, categorizing participants' granzyme B

220 responses as either increasing, decreasing, or not changing revealed heterogeneity (Fig. S2),

221 though proportionally there were fewer decreasing responses, and the population-average levels

222 of granzyme B responses were highly stable over time to all HIV-gene products (Fig. 5B and

223 Table S4). In contrast to IFN- $\gamma$, we did not observe any significant correlations between the slopes

224 of change of granzyme B responses with virologic measures of HIV persistence (Tables S9 \&

225 S11). These results may reflect the additional complexity that whereas both IFN- $\gamma$ - and granzyme

226 B-producing cells can be maintained by infected cells producing antigen, the latter are more likely

227 to also perturb the virologic measures by eliminating infected cells (37).

228 To further assess the functional profiles of HIV-specific T-cell responses, we performed

229 pairwise comparisons of granzyme B versus IFN- $\gamma$ responses for each of the gene products tested

230 (Fig. 5C). At both timepoints, granzyme B response magnitudes to Gag, Pol, and CMV-pp65 were

231 substantially lower than IFN- $\gamma$ responses (all $p<0.05)$ (Fig. 5C). Contrasting this, the magnitudes

232 of granzyme B versus IFN- $\gamma$ responses to Nef were not significantly different from each other at

233 either timepoint $(p=0.100$ at week $24, p=0.277$ at week 168). These data indicate that in addition

234 to being preferentially maintained over time, T-cell responses directed against the early HIV gene

235 product Nef disproportionately exhibit effector functional profiles, as compared to the late gene

236 products Gag and Pol (though appreciable granzyme B responses to these gene products were 
237 still detected). Persistent HIV-specific granzyme B responses are indicative of recent antigen

238 encounter, supporting the hypothesis that there is in vivo stimulation by HIV-infected cells despite

239 suppressive ART.

\section{Discussion}

An important aspect of how HIV persists in individuals on long-term ART is through the

242 evasion of immune recognition, predominately thought to be achieved through the maintenance

243 of strict viral latency, with an additional aspect of anatomical sequestration. This perception that

244 the reservoir is entirely latent has begun to shift lately, in response both to a new understanding

245 of the dynamic nature of the HIV reservoir (driven by the clonal expansion of infected cells), and

246 to new insights into ongoing viral transcriptional activity on ART $(15,38)$. To date, however, this

247 has yet to prompt widespread re-consideration of the relationship between the HIV-specific T-cell

248 response and the HIV reservoir. The current study provides evidence which challenges the

249 prevailing model of a lack of reservoir immune surveillance, by indicating a level of ongoing

250 antigenic stimulation of HIV-specific T-cells in ART-suppressed individuals. Nef-specific T-cells

251 stood apart from those of other HIV gene products in this regard, supporting that early gene

252 products (Nef, Tat, and Rev - of which only Nef was appreciably immunogenic [as also seen in

253 other studies $(21,33)])$ have lower thresholds to expression in a reactivation setting as compared

254 to late gene products (Gag, Pol, and Env), which are expressed only after a cell has built up

255 sufficient levels of Rev to drive nuclear export of unspliced and singly-spliced viral transcripts (39,

256 40). The preferential maintenance of Nef-specific T-cells was presented as a hypothesis of the

257 current study based, in part, on our previous observation that Nef-specific T-cells recognized cells

258 reactivated from an in vitro latency model prior to recognition by Gag-specific T-cells, or

259 detectable Gag expression (32). 
Do our results allow for any inferences into how frequently infected cells are recognized by HIV-specific T-cells in vivo? While numerous aspects of complexity introduce caveats to such an analysis (e.g. tissue distributions), our data do allow for side-by-side comparisons between the peripheral blood frequencies of infected cells with antigen-expression potential, and those of HIV-

264 specific T-cells - which may be informative. The median frequency of Nef-specific T-cells at week 26524 of our study was $78.5 / 10^{6}$ PBMCs, whereas the median total frequency of HIV-infected cells 266 (CA-DNA) was 515.7/10 CD4 $^{+}$T-cells (at week 0), or roughly $103 / 10^{6}$ PBMCs. These infected 267 cells, however, predominately contain defective proviruses (41), many of which are likely 268 incapable of expressing antigens (42). It can therefore be reasonably estimated that, in most 269 individuals, Nef-specific T-cells are at least as frequent as infected cells with the potential to 270 express antigen. Our data indicating that the former are influenced by the latter therefore suggest 271 that antigen expression is more likely to be a common versus a rare event in vivo, amongst 272 infected cells with this potential. Further study is needed, however, and characterizing the clonal 273 dynamics of HIV-specific T-cells may yield additional insights.

Although latency almost certainly contributes to viral persistence, our findings indicating that

275 HIV reservoirs are not fully hidden from circulating cytotoxic T-cells raise the question of what 276 additional mechanisms may be at play. We first consider the role of immune escape - the process 277 by which HIV evades recognition by acquiring mutations in T-cell epitopes. Immune escape plays 278 a critical role in limiting the overall efficacy of the HIV-specific T-cell response in untreated 279 infection, and HIV reservoirs show clear evidence of past selection, in the form of extensive 280 sequence variation in known T-cell epitopes (29). However, the question at hand pertains to HIV281 specific T-cell responses that show evidence of being maintained by recent antigen recognition, 282 indicating that they target epitopes which are intact in at least a portion of the reservoir. Further 283 supporting this idea are the previous observations that: i) the fixation of escape mutations leads 284 to the contraction of corresponding T-cell responses (43), and ii) the substantial majority of HIV- 
285 specific T-cells that remain detectable after years of ART target epitopes for which escape is not

286 fixed in corresponding reservoir viruses $(44,45)$. As with latency, our data do not lead us to 287 contest the idea that the fixation of escape mutations in the reservoir diminishes the overall 288 potential for immune recognition, nor the value of therapeutic strategies to address either of these 289 limitations. However, we are still left with the question of how to reconcile our findings indicating 290 an appreciable level of ongoing in vivo recognition of infected cells by cytotoxic (granzyme B) T291 cells, with the overall stability of HIV reservoir sizes.

We therefore draw from two recent findings in the field to propose how an HIV reservoir may 293 persist without being fully hidden from circulating cytotoxic T-cells. The first derives from the 294 recent demonstrations that the HIV reservoir is predominately composed of infected T-cells that 295 have undergone clonal expansion (46-48), with different clones dynamically 'waxing and waning' 296 over time (48). Thus, HIV-specific T-cells may frequently eliminate infected cells, only to have 297 these replaced by clonal expansion of other reservoir-harboring cells. There have been somewhat 298 conflicting recent reports regarding this possibility - from groups that approached the question 299 from different angles - highlighting the need for further study $(42,49,50)$.

301 to T-cell mediated elimination (51), mediated in part by BCL-2 over-expression, which

302 antagonizes perforin/granzyme killing (52). In fact, while it has been generally assumed in our

303 field that the encounter between an antigen-expressing HIV-infected cell and a functional (ex.

304 perforin/granzyme releasing) $\mathrm{CD}^{+}$T-cell will result in elimination, this overlooks the role of the

305 target cell as an active partner in the killing process. Multiple regulatory mechanisms exist, both 306 in physiological and pathological states, by which target cells determine whether or not to undergo 307 apoptosis, despite receiving a perforin/granzyme hit $(53,54)$. Thus, one way to resolve our 308 findings with others in the field is to propose that the recognition of HIV-infected cells by HIV- 
309 specific cytotoxic T-cells may occur with some frequency in vivo, but that this often does not result

310 in target cell elimination. An intriguing possibility is that the combined effects of selection, based

311 on intrinsic susceptibility to $\mathrm{CD}^{+} \mathrm{T}$-cells, and clonal expansion of surviving cells may enable the

312 evolution of a resistant reservoir, paralleling the phenomenon of 'immunoediting' in cancer (11).

313 While latency reversal will likely be a critical component of curing HIV infection, our findings raise

314 the hypothesis that - in lieu of an ideal latency reversing agent - reductions in HIV reservoirs may

315 be achievable by boosting immune targeting of existing expression of early gene products (such

316 as Nef, and in a manner that targets non-escaped epitopes,) while enhancing cytotoxic function,

317 limiting clonal expansion, and addressing resistance to cytotoxic T-cells in reservoir-harboring 318 cells.

319 Methods

320 Study Design

321 For these observational studies, we evaluated participants from two separate populations. The 322 data in Fig. 1 were collected on participants diagnosed with HIV infection recruited via 323 convenience sampling through Maple Leaf Medical Clinic in Toronto, Canada. Outliers were not 324 defined or excluded. Participants in this Toronto cohort were virally suppressed for a minimum of 3252 years prior to a leukapheresis procedure to collect PBMCs, with no reported ART interruptions 326 or detectable viral loads by a commercial clinical assay. The objective of this first study was to 327 evaluate the total ability of participant's $\mathrm{CD}^{+}$T-cells to recognize autologous HIV reservoir 328 viruses, and to compare these results with T-cell responses as measured by IFN-y ELISPOT. All 329 other data for this manuscript were collected on a longitudinal cohort of participants who initiated 330 ART during chronic HIV infection in AIDS Clinical Trials Group (ACTG) trials for treatment-naïve 331 individuals, and enrolled in the ACTG HIV Reservoirs Cohort Study (A5321) (31). A5321 cohort 332 participants were recruited from 17 clinical research sites in the United States through the ACTG 
333 network. IFN-y ELISPOTs were previously performed using samples from 96 participants at

334 A5321 study entry (32), and a subset of 49 participants were selected from the original 96 for this

335 longitudinal sub-study based on sample availability. All gene products and negative controls were

336 tested in duplicate, with one replicate of PHA positive control. Assays performed under these

337 same conditions have been previously validated in other participant cohorts. Outliers were not

338 defined or excluded. Participants in the current sub-study had follow-up at least every 6 months

339 following study entry, with documented sustained viral suppression (plasma HIV RNA levels $<50$

340 copies/mL by commercial assays starting at week 48 on ART and at all subsequent timepoints -

341 Fig. 2). One participant had a large viral blip (>1,000 copies $/ \mathrm{mL}) 43$ weeks prior to their 168 week

342 A5321 study timepoint, and data was right-censored for this participant after the 24 week A5321

343 study timepoint. Clinical data and paired plasma and PBMC samples were available from pre-

344 ART and on ART study visits. We measured HIV levels (CA-DNA, CA-RNA, and plasma iSCA)

345 and PD-1 levels (on $\mathrm{CD}^{+}$and $\mathrm{CD}^{+}$cells) on samples obtained at A5321 study entry (median 7

346 years on ART), and plasma HIV RNA levels and CD4 ${ }^{+}$T-cell counts were obtained from pre-ART

347 clinical data. One participant later revoked consent for further testing and was excluded from

348 analysis. We hypothesized a priori that the long-term dynamics of T-cell responses to the early

349 HIV gene product Nef (measured by IFN-y ELISPOT) would be associated with infected cell

350 frequencies.

351 Virologic Assays

352 HIV CA-DNA and CA-RNA were measured by quantitative PCR (qPCR) assays in PBMCs using 353 previously described methods (55). CA-DNA and CA-RNA values per million CD4 ${ }^{+}$T-cells were 354 calculated by dividing the total CA-DNA or CA-RNA copies/million PBMCs (normalized for CCR5 355 copies measured by qPCR as published (55)) by the CD4 ${ }^{+}$T-cell percentage $(x 0.01)$ reported 356 from the same specimen date or from a CD4 ${ }^{+} \mathrm{T}$-cell percentage imputed using linear interpolation 
357 from specimen dates before and after the CA-DNA or CA-RNA results. Cell-free HIV RNA was 358 quantified by iSCA in blood plasma $(5 \mathrm{~mL})(56)$.

359

\section{Immunologic Assays}

PBMCs obtained at A5321 study entry were stained with the following monoclonal antibodies to evaluate surface PD-1 expression: CD3 APC-H7, CD4 PC5, CD8 V450, PD-1 (clone M1H4) A488 (all from BD Biosciences, San Diego, California, USA), and Live/Dead Aqua (Invitrogen, Grand Island, New York, USA). Cells were fixed in 1\% paraformaldehyde, and analyzed using a BD LSR Fortessa (FACSDiva) within 24 hours after staining. Lymphocytes were identified based upon size and granularity. The lymphocyte population was filtered through side scatter area vs. side scatter height histogram to eliminate doublets from the analysis. Single cells were analyzed using Live/Dead Aqua dye exclusion and then $\mathrm{CD}^{+}$and $\mathrm{CD}^{+}$populations were defined based on dual expression with CD3. These two populations were plotted against PD-1. Fluorescence minus one (FMO) controls were used to define the PD-1 $1^{+}$T-cell populations.

\section{Quantitative Viral Outgrowth Assay (QVOA)}

Quantitative Viral Outgrowth Assays (QVOA) were performed as previously described (57). Briefly, CD4 ${ }^{+}$T-cells were isolated from PBMCs by negative selection (Easysep, Stemcell Technologies) and plated in serial dilution at either 4 or 6 concentrations (12 wells/concentration, 24-well plates). $\mathrm{CD}^{+}$T-cells were stimulated with phytohemagglutinin $(\mathrm{PHA}, 2 \mu \mathrm{g} / \mathrm{mL})$ and irradiated allogeneic HIV-negative PBMCs were added to further induce viral reactivation. MOLT4/CCR5 cells were added at 24 hours post-stimulation as targets for viral infection. Culture media [RPMI $1640+10 \%$ FBS + 1\% Pen/Strep +50U/mL IL-2 + 10ng/mL IL-15 (R10-50-15)] was changed every 3 days and p24 enzyme-linked immunosorbent assay (ELISA, NCI Frederick) was run on day 14 to identify virus-positive wells. Infectious Units per Million CD4 ${ }^{+}$T-cells (IUPM) values were determined using the Extreme Limiting Dilution Analysis (ELDA) software 
381 (http://bioinf.wehi.edu.au/software/elda/) (58). Culture supernatants from virus-positive wells were

382 frozen $\left(-80^{\circ} \mathrm{C}\right)$ for future use.

384 Activation of CD4 ${ }^{+}$T-cell Targets: CD4 ${ }^{+}$T-cells were enriched by negative selection (Easysep,

385 Stemcell Technologies), typically starting from $200 \times 10^{6}$ PBMCs per study participant. These cells

386 were stimulated with $10 \mu \mathrm{g} / \mathrm{ml}$ of anti-CD3 (OKT-3) and anti-CD28 (28.2) antibodies (Ultra-LEAF ${ }^{\mathrm{TM}}$,

387 Biolegend) in R10-50-15 for 48 hours. Infections: Cells were then washed, and split into 3 equal

388 aliquots $\left(\sim 4 \times 10^{6}\right.$ cells each) for infection with either: i) JRCSF ii) autologous virus or iii) mock

389 (nothing). The autologous virus stock was generated by pooling equal volumes of all p24 ${ }^{+} \mathrm{QVOA}$

390 wells from that study participant, while the JRCSF stock was generated by transfection of 293T

391 cells with plasmid. All viruses were titrated on TZM-bl cells, and used at a MOI of 0.4. After a 2

392 hour infection period at $37^{\circ} \mathrm{C} 5 \% \mathrm{CO}_{2}$, cells were washed and then cultured for 48 hours in R10-

393 50. Levels of infection were monitored every 24 hours by surface staining small aliquots with anti-

394 CD3 Brilliant Violet $785^{\mathrm{TM}}$, CD4 Pacific Blue ${ }^{\mathrm{TM}}$ (Biolegend), then permeabilizing (BD

395 Cytofix/Cytoperm ${ }^{\mathrm{TM}}$ ) and staining intracellularly with anti-HIV-Gag Kc57-RD1 (Beckman Coulter),

396 and analyzed on a BD LSRFortessa ${ }^{\text {TM }}$ flow cytometer. Infections were harvested for co-culture

397 with $\mathrm{CD}^{+} \mathrm{T}$-cells, when they reached $2-4 \% \mathrm{Gag}^{+}$within the $\mathrm{CD}^{+}$gate.

398 Co-culture with $\mathrm{CD}^{+}$T-cells: $\mathrm{CD}^{+}{ }^{+}$T-cells autologous to these $\mathrm{CD} 4^{+}$targets were enriched on

399 the day of co-culture from freshly a thawed aliquot of $100 \times 10^{6}$ PBMCs by negative selection

400 (Easysep ${ }^{\mathrm{TM}}$, Stemcell Technologies). Infected and mock-infected CD4 ${ }^{+}$T-cells were washed $5 \mathrm{x}$

401 and then co-cultured with $\mathrm{CD}^{+} \mathrm{T}$-cells at ratios of $5 \mathrm{CD}^{+} \mathrm{T}$-cells to $1 \mathrm{CD}^{+} \mathrm{T}^{-}$-cell, at a total

402 concentration of $5 \times 10^{6}$ cells/mL in RPMI $1640+10 \%$ FBS $+1 \%$ Pen/Strep $+50 U / m L$ IL-2 (R10-

403 50) with 1/100 anti-CD107a-PE antibody (Biolegend) and 1/1,000 Monensin GolgiStop ${ }^{\mathrm{TM}}$ (BD).

404 Cells were incubated for a total of 6 hours, with mixing by pipetting every 30 minutes (to facilitate 
405 contacts between antigen-specific $\mathrm{CD}^{+}$cells and targets). Staining and Analysis: Cells were 406 surface stained with anti-CD3 Brilliant Violet $785^{\mathrm{TM}}$, CD4 Pacific Blue ${ }^{\mathrm{TM}}$, CD8 Alexa Fluor ${ }^{\circledR} 700$, 407 and LIVE/DEAD ${ }^{\text {TM }}$ Fixable Aqua dye (Thermofisher). Cells were then washed, permeabilized (BD 408 Cytofix/Cytoperm ${ }^{\mathrm{TM}}$ ), stained intracellularly with anti-HIV-Gag Kc57-RD1 (Beckman Coulter), and 409 analyzed on a BD LSRFortessa ${ }^{\text {TM }}$ flow cytometer.

\section{Peptide Pools}

411 The following sets of consensus HIV clade B 15 amino acid peptides (overlapping by 11 amino

412 acids) were supplied by the NIH AIDS Research and Reference Reagent Program: Gag (cat \# 413 8117), Env (cat\# 9480), Pol (cat \# 6208), Tat (cat\# 5138), Rev (cat\# 6445), and Nef (cat \# 5189).

414 All peptides were dissolved at $5 \mathrm{mg} / \mathrm{mL}$ in 12.5\% DMSO (Corning), and 87.5\% PBS (Gibco). 415 Peptides were pooled into whole gene product peptide pools and adjusted to a final concentration 416 of $20 \mu \mathrm{g} / \mathrm{mL} /$ peptide in PBS. A CMV-pp65 PepMix peptide pool (JPT Peptide Technologies) was 417 dissolved separately in DMSO and adjusted to a final concentration of $20 \mu \mathrm{g} / \mathrm{mL} / \mathrm{peptide}$ in PBS.

418 IFN-y and Granzyme B ELISPOT Assays

419 Multi-screen IP 96-well PVDF plates (Millipore) were either directly coated with 100 $\mu$ L/well of PBS $420+0.5 \mu \mathrm{g} / \mathrm{mL}$ primary anti-human IFN-y antibody (clone $1-\mathrm{D} 1 \mathrm{~K}$, Mabtech) overnight at $4^{\circ} \mathrm{C}$, or first 421 primed with $20 \mu \mathrm{L}$ of $35 \% \mathrm{EtOH} /$ well, and immediately washed $6 \mathrm{x}$ with $200 \mu \mathrm{L} \mathrm{ddH}_{2} \mathrm{O}$ and then 422 coated with $100 \mu \mathrm{L} /$ well of PBS $+15 \mu \mathrm{g} / \mathrm{mL}$ primary anti-human granzyme B antibody (clone GB10, 423 Mabtech) overnight at $4^{\circ} \mathrm{C}$. Granzyme B plates were washed $6 x$ with $200 \mu \mathrm{L}$ PBS and blocked 424 with RPMI 10\% FBS (Gibco) ('R-10') at $37^{\circ} \mathrm{C} 5 \% \mathrm{CO}_{2}$. PBMCs were thawed and resuspended in

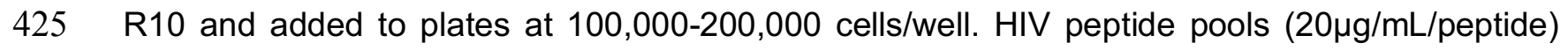
426 were added at $10 \mu \mathrm{L} /$ well for a final concentration of $1 \mu \mathrm{g} / \mathrm{mL} /$ peptide in $<0.5 \%$ DMSO. CMV-pp65 427 peptide pools were added at $10 \mu \mathrm{L} /$ well for a final concentration of $1 \mu \mathrm{g} / \mathrm{mL} /$ peptide in $<0.5 \%$ 428 DMSO. PHA was dissolved in DMSO and PBS to $200 \mu \mathrm{g} / \mathrm{mL}$, and then added to a final 
concentration of $1 \mu \mathrm{g} / \mathrm{mL}$ as a positive control. $0.5 \%$ DMSO in PBS and R-10 media were used as

430 negative controls. Plates were incubated for 18 hours at $37^{\circ} \mathrm{C}$ with $5 \% \mathrm{CO}_{2}$. Plates were washed

431 6x with $200 \mu \mathrm{L}$ PBS. Biotinylated secondary IFN-ץ antibody (clone 7-B6-1, Mabtech) at $0.5 \mu \mathrm{g} / \mathrm{mL}$

432 in PBS, or biotinylated secondary anti-granzyme B antibody (clone GB11, Mabtech) at $1.0 \mu \mathrm{g} / \mathrm{mL}$

433 in PBS was added to the plates to a final volume of $100 \mu \mathrm{L}$ and incubated for 1 hour in the dark.

434 Plates were then washed $6 \mathrm{x}$ with PBS and $0.5 \mu \mathrm{g} / \mathrm{mL}$ of Streptavidin-ALP (Mabtech) was added

435 to IFN- $\gamma$ plates at $100 \mu \mathrm{L} /$ well, and $1 \mu \mathrm{g} / \mathrm{mL}$ of Streptavidin-ALP (Mabtech) was added to granzyme

436 B plates at $100 \mu \mathrm{L} /$ well and incubated for 1 hour. Plates were washed $6 \mathrm{x}$ with PBS and then color

437 development substrate solution: $10.6 \mathrm{~mL}$ of $\mathrm{ddH}_{2} \mathrm{O}, 400 \mu \mathrm{L} 25 x$ AP Color Development Buffer

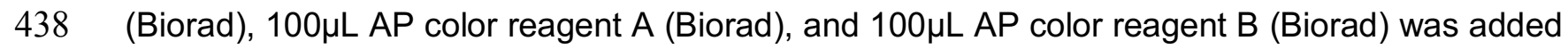

439 to the plate at $100 \mu \mathrm{L} /$ well for 15 minutes. After removal of the color development substrate

440 solution, $0.5 \%$ of Tween-20 in PBS was added at $100 \mu \mathrm{L} /$ well for 10 minutes. Plates were then

441 washed with water, and left overnight to dry. Plates were counted using Immunospot S6 Ultimate

442 Analyzer and ImmunoSpot software (Cellular Technology Limited).

\section{Statistics}

444 Statistical analyses including univariate statistics and Spearman $r$ correlations and partial 445 correlations (adjusting for potential confounders) were conducted in SAS University Edition.

446 Slopes of change in Fig. 4B and Tables S5-S6 and S9-S10 were calculated based on absolute

447 changes on a linear scale between weeks 24-168 post-A5321 study entry, excluding participants

448 who had a change from 0 magnitude to 0 magnitude. Analyses for Tables S7-S8 and S11-S12

449 used slopes of change calculated based on proportional changes on a $\log _{10}$ scale between weeks

450 24-168 post-A5321 study entry, excluding participants who had a change from 0 magnitude to 0

451 magnitude; slopes reflecting a change from 0 magnitude to a non-zero magnitude were analyzed

452 as the highest rank, and slopes reflecting a change from a non-zero magnitude to 0 magnitude

453 were analyzed as the lowest rank. Statistical analyses including one-way ANOVA and Wilcoxon 
454 signed-rank tests were conducted in GraphPad Prism v.8.0. Plots for figures were made in

455 GraphPad Prism v.8.0 and SAS University Edition. A custom code was generated in MATLAB

456 v.9.7 to produce the correlogram in Fig. 4B. All linear mixed-effects models were conducted using

457 the R 'Ime4' package (59), with random intercepts only or both random intercepts and random

458 slopes on the participant level modeled for the random effects, as assessed by significantly

459 improved model fit when random slopes were included (using the R ANOVA function to compare

460 models); multiple comparisons were made where indicated using the R 'multcomp' package (60),

461 adjusting for multiple comparisons using Tukey's all-pair method. Linear mixed-effects models

462 used $\log _{10}$-transformed response data, treating zero-valued responses as missing data.

463 Imputation was not used to address missing data, as the degree of missingness was low. All

464 statistical tests were two-sided, $\alpha=0.05$.

465 Study Approval

466 Ethics oversight for part 1 of this study, for participants from Maple Leaf Medical Clinic, was

467 provided by The George Washington University under IRB protocol \#021750. Participants were

468 recruited via convenience sampling by research staff at Maple Leaf Medical Clinic during routine

469 care visits; prospective participants were provided a verbal description of the research and a copy

470 of the informed consent form, which detailed the study's objectives, risks, and benefits. For part

4712 of this study, each ACTG A5321 clinical research site had the A5321 protocol and consent form,

472 and its relevant parental protocols and consent forms, approved by their local IRB, as well as

473 registered with and approved by the Division of AIDS (DAIDS) Regulatory Support Center (RSC)

474 Protocol Registration Office, prior to any participant recruitment and enrollment. Once a

475 participant for study entry was identified, details were carefully discussed with the prospective

476 participant by clinical staff at the site. The participant (or, when necessary, the parent or legal

477 guardian if the participant was under guardianship) was asked to read and sign the ACTG-

478 approved protocol consent form. 
480 Author contributions: RBJ designed the study. EMS, ARW, RT, AST, SHH, TRD, ST, JKB,

481 TMM, AD, GQL, AG, PK, WDCA, JCC, and BM performed experiments. EMS, ARW, RT, ST,

$482 \mathrm{SHH}, \mathrm{JKB}, \mathrm{CML}, \mathrm{RJB}, \mathrm{BM}, \mathrm{JCC}$, and JWM analyzed data. RTG, DKM, JJE, and JWM provided

483 participant data. EMS, ARW, and RBJ wrote the manuscript. All authors contributed to the

484 critical revision of the manuscript.

Acknowledgments: This work was supported by 1) the Martin Delaney 'BELIEVE'

Collaboratory (NIH grant 1UM1AI26617), which is supported by the following NIH Co-Funding and Participating Institutes and Centers: NIAID, NCI, NICHD, NHLBI, NIDA, NIMH, NIA, FIC, and OAR; 2) by the National Institute of Allergy and Infectious Diseases of the National Institutes of Health under Award Number UM1 AI068634, UM1 AI068636 and UM1 Al106701; and 3) by a grant from the AIDS Clinical Trials Group Network (ACTG) to the University of

492 Pittsburgh Virology Specialty Laboratory. It was also supported in part by the NIH funded R01

493 grants Al31798 and Al147845, and by an ACTG special projects grant (to RBJ). We thank Shy

494 Genel for assistance with MATLAB coding. We gratefully acknowledge the contributions of the

495 study participants, without whom this work would not be possible. The content is solely the

496 responsibility of the authors and does not necessarily represent the official views of the National

497 Institutes of Health.

498 JWM is a consultant to Gilead Sciences and Merck, and owns share options in Co-Crystal 499 Pharmaceuticals and Abound Bio, Inc., which are not involved in the current work. JJE has 500 research funding outside the current work from ViiV Healthcare, Gilead Sciences and Janssen, 501 and has consulting income from ViiV Healthcare, Gilead Sciences, Janssen, and Merck. BM has 502 received research funding from Gilead Sciences. RTG has served on a scientific advisory board 503 for Merck. EMS volunteers on the Board of Directors of the non-profit community clinic the 
504 Berkeley Community Health Project. The authors declare that they have no other perceived

505 conflicts of interest.

\section{References}

508 1. UNAIDS. Global HIV and AIDS statistics - 2019 fact sheet [Internet]. FACT SHEET - WORLD

509 AIDS DAY 2019; https://www.unaids.org/en/resources/fact-sheet.

510 2. Andrade VM et al. A minor population of macrophage-tropic HIV-1 variants is identified in 511 recrudescing viremia following analytic treatment interruption. Proceedings of the National 512 Academy of Sciences 2020;117(18):9981-9990.

513 3. Ganor Y et al. HIV-1 reservoirs in urethral macrophages of patients under suppressive 514 antiretroviral therapy. Nature Microbiology 2019;4(4):633-644.

515 4. Finzi D et al. Identification of a Reservoir for HIV-1 in Patients on Highly Active Antiretroviral 516 Therapy. Science 1997;278(5341):1295-1300.

517 5. Chun TW et al. Presence of an inducible HIV-1 latent reservoir during highly active 518 antiretroviral therapy. Proceedings of the National Academy of Sciences of the United States of 519 America 1997;94(24):13193-13197.

520 6. Wong JK et al. Recovery of Replication-Competent HIV Despite Prolonged Suppression of 521 Plasma Viremia. Science 1997;278(5341):1291-1295.

522 7. Siliciano JD, Siliciano RF. Enhanced Culture Assay for Detection and Quantitation of Latently 523 Infected, Resting CD4+ T-Cells Carrying Replication-Competent Virus in HIV-1-Infected 524 Individuals. In: Human Retrovirus Protocols. New Jersey: Humana Press; 2005:003-016

525 8. Siliciano JD et al. Long-term follow-up studies confirm the stability of the latent reservoir for 526 HIV-1 in resting CD4+ T cells. Nature Medicine 2003;9(6):727-728.

527 9. Castagna A et al. Analytical treatment interruption in chronic HIV-1 infection: time and 528 magnitude of viral rebound in adults with 10 years of undetectable viral load and low HIV-DNA 529 (APACHE study). Journal of Antimicrobial Chemotherapy 2019;74(7):2039-2046.

530 10. Deeks SG. Shock and kill. Nature 2012;487(7408):439-440.

531 11. Huang S-H et al. Have Cells Harboring the HIV Reservoir Been Immunoedited?. Frontiers in 532 Immunology 2019;10. doi:10.3389/fimmu.2019.01842

533 12. Yukl SA et al. HIV latency in isolated patient CD4 + T cells may be due to blocks in HIV 534 transcriptional elongation, completion, and splicing. Science Translational Medicine 535 2018;10(430):eaap9927.

536 13. Fischer $M$ et al. Cellular Viral Rebound after Cessation of Potent Antiretroviral Therapy 537 Predicted by Levels of Multiply Spliced HIV-1 RNA Encoding nef. The Journal of Infectious 538 Diseases 2004;190(11):1979-1988. 
14. Pace MJ, Agosto L, Graf EH, O’Doherty U. HIV reservoirs and latency models. Virology 2011;411(2):344-354.

541 15. Cohn LB, Chomont N, Deeks SG. The Biology of the HIV-1 Latent Reservoir and

542 Implications for Cure Strategies. Cell Host \& Microbe 2020;27(4):519-530.

543 16. Mota TM et al. Integrated Assessment of Viral Transcription, Antigen Presentation, and CD8 + T Cell Function Reveals Multiple Limitations of Class I-Selective Histone Deacetylase Inhibitors during HIV-1 Latency Reversal. Journal of Virology 2020;94(9). doi:10.1128/JVI.01845-19

17. Jones RB et al. A Subset of Latency-Reversing Agents Expose HIV-Infected Resting CD4+ T-Cells to Recognition by Cytotoxic T-Lymphocytes. PLOS Pathogens 2016;12(4):e1005545.

18. Casazza JP, Betts MR, Picker LJ, Koup RA. Decay Kinetics of Human Immunodeficiency Virus-Specific CD8+ T Cells in Peripheral Blood after Initiation of Highly Active Antiretroviral Therapy. Journal of Virology 2001;75(14):6508-6516.

19. Kalams SA et al. Levels of human immunodeficiency virus type 1-specific cytotoxic Tlymphocyte effector and memory responses decline after suppression of viremia with highly active antiretroviral therapy. Journal of Virology 1999;73(8):6721-6728.

20. Ogg GS et al. Decay Kinetics of Human Immunodeficiency Virus-Specific Effector Cytotoxic

T Lymphocytes after Combination Antiretroviral Therapy. Journal of Virology 1999;73(1):797800.

21. Xu Y et al. HIV-Specific T Cell Responses Are Highly Stable on Antiretroviral Therapy. Molecular Therapy - Methods \& Clinical Development 2019;15:9-17.

560 22. Rouzioux C, Avettand-Fenoël V. Total HIV DNA: a global marker of HIV persistence.

561 Retrovirology 2018;15(1):30.

562 23. Imamichi H et al. Defective HIV-1 proviruses produce novel protein-coding RNA species in

563 HIV-infected patients on combination antiretroviral therapy. Proceedings of the National

564 Academy of Sciences 2016;113(31):8783-8788.

565 24. Wolint $P$, Betts MR, Koup RA, Oxenius A. Immediate Cytotoxicity But Not Degranulation

566 Distinguishes Effector and Memory Subsets of CD8+ T Cells. Journal of Experimental Medicine

567 2004;199(7):925-936.

568 25. Shin H, Blackburn SD, Blattman JN, Wherry EJ. Viral antigen and extensive division 569 maintain virus-specific CD8 T cells during chronic infection. Journal of Experimental Medicine $570 \quad 2007 ; 204(4): 941-949$.

571 26. Rock MT et al. Differential Regulation of Granzyme and Perforin in Effector and Memory T 572 Cells following Smallpox Immunization. The Journal of Immunology 2005;174(6):3757-3764.

573 27. Roberts ER et al. Collapse of Cytolytic Potential in SIV-Specific CD8+ T Cells Following 574 Acute SIV Infection in Rhesus Macaques. PLOS Pathogens 2016;12(12):e1006135. 
28. McElhaney JE et al. Granzyme B: Correlates with protection and enhanced CTL response to influenza vaccination in older adults. Vaccine 2009;27(18):2418-2425.

29. Deng $\mathrm{K}$ et al. Broad CTL response is required to clear latent HIV-1 due to dominance of escape mutations. Nature 2015;517(7534):381-385.

30. Sewell AK et al. IFN-gamma exposes a cryptic cytotoxic T lymphocyte epitope in HIV-1 reverse transcriptase. Journal of Immunology 1999;162(12):7075-7079.

31. Gandhi RT et al. Levels of HIV-1 persistence on antiretroviral therapy are not associated with markers of inflammation or activation. PLOS Pathogens 2017;13(4):e1006285.

32. Thomas AS et al. T-cell responses targeting HIV Nef uniquely correlate with infected cell frequencies after long-term antiretroviral therapy. PLOS Pathogens 2017;13(9):e1006629.

33. Addo MM et al. Comprehensive Epitope Analysis of Human Immunodeficiency Virus Type 1 (HIV-1)-Specific T-Cell Responses Directed against the Entire Expressed HIV-1 Genome Demonstrate Broadly Directed Responses, but No Correlation to Viral Load. Journal of Virology 2003;77(3):2081-2092.

34. Nowacki TM et al. Granzyme B production distinguishes recently activated CD8+ memory cells from resting memory cells. Cellular Immunology 2007;247(1):36-48.

35. Migueles SA et al. Lytic Granule Loading of CD8+ T Cells Is Required for HIV-Infected Cell Elimination Associated with Immune Control. Immunity 2008;29(6):1009-1021.

36. Shafer-Weaver $\mathrm{K}$ et al. The Granzyme B ELISPOT assay: an alternative to the $51 \mathrm{Cr}$-release assay for monitoring cell-mediated cytotoxicity. Journal of Translational Medicine 2003;1(1):14.

37. Yue FY et al. HIV-Specific Granzyme B-Secreting but Not Gamma Interferon-Secreting T Cells Are Associated with Reduced Viral Reservoirs in Early HIV Infection. Journal of Virology 2017;91(8). doi:10.1128/JVI.02233-16

38. Pasternak AO, Berkhout B. What do we measure when we measure cell-associated HIV RNA. Retrovirology 2018;15(1):13.

39. Felber BK, Hadzopoulou-Cladaras M, Cladaras C, Copeland T, Pavlakis GN. Rev protein of human immunodeficiency virus type 1 affects the stability and transport of the viral mRNA. Proceedings of the National Academy of Sciences 1989;86(5):1495-1499.

40. Malim MH, Hauber J, Le S-Y, Maizel J v., Cullen BR. The HIV-1 rev trans-activator acts through a structured target sequence to activate nuclear export of unspliced viral mRNA. Nature 1989;338(6212):254-257.

41. Ho Y-C et al. Replication-Competent Noninduced Proviruses in the Latent Reservoir Increase Barrier to HIV-1 Cure. Cell 2013;155(3):540-551.

42. Pollack RA et al. Defective HIV-1 Proviruses Are Expressed and Can Be Recognized by Cytotoxic T Lymphocytes, which Shape the Proviral Landscape. Cell Host \& Microbe 2017;21(4):494-506.e4. 
611

612

613

614

615

616

617

618

619

620

621

622

623

624

625

626

627

628

629

630

631

632

633

634

635

636

637

638

639

640

641

642

643

644

645

43. Allen TM et al. De Novo Generation of Escape Variant-Specific CD8+ T-Cell Responses following Cytotoxic T-Lymphocyte Escape in Chronic Human Immunodeficiency Virus Type 1 Infection. Journal of Virology 2005;79(20):12952-12960.

44. Warren $\mathrm{J}$ et al. HIV-1 in the latent reservoir is largely sensitive to circulating $\mathrm{T}$ cells. Journal of Virus Eradication 2019;5(Supplement 3):PP 5.7.9.

45. Veenhuis RT et al. Long-term remission despite clonal expansion of replication-competent HIV-1 isolates. JCI Insight 2018;3(18). doi:10.1172/jci.insight.122795

46. Lee GQ et al. Clonal expansion of genome-intact HIV-1 in functionally polarized Th1 CD4+ T cells. Journal of Clinical Investigation 2017;127(7):2689-2696.

47. Cohn LB et al. HIV-1 Integration Landscape during Latent and Active Infection. Cell 2015;160(3):420-432.

48. Wang Z et al. Expanded cellular clones carrying replication-competent HIV-1 persist, wax, and wane. Proceedings of the National Academy of Sciences 2018;115(11):E2575-E2584.

49. Pinzone MR et al. Longitudinal HIV sequencing reveals reservoir expression leading to decay which is obscured by clonal expansion. Nature Communications 2019;10(1):728.

50. Antar AA et al. Longitudinal study reveals HIV-1-infected CD4+ T cell dynamics during longterm antiretroviral therapy. Journal of Clinical Investigation 2020;130(7):3543-3559.

51. Knapp DJHF et al. Increasingly Successful Highly Active Antiretroviral Therapy Delays the Emergence of New HLA Class I-Associated Escape Mutations in HIV-1. Clinical Infectious Diseases 2012;54(11):1652-1659.

52. Ren $Y$ et al. BCL-2 antagonism sensitizes cytotoxic T cell-resistant HIV reservoirs to elimination ex vivo. Journal of Clinical Investigation 2020;130(5):2542-2559.

53. Medema JP et al. Expression of the Serpin Serine Protease Inhibitor 6 Protects Dendritic Cells from Cytotoxic T Lymphocyte-Induced Apoptosis. The Journal of Experimental Medicine 2001;194(5):657-668.

54. Patel SJ et al. Identification of essential genes for cancer immunotherapy. Nature 2017;548(7669):537-542.

55. Hong F et al. Novel Assays for Measurement of Total Cell-Associated HIV-1 DNA and RNA. Journal of Clinical Microbiology 2016;54(4):902-911.

56. Cillo AR et al. Improved Single-Copy Assays for Quantification of Persistent HIV-1 Viremia in Patients on Suppressive Antiretroviral Therapy. Journal of Clinical Microbiology 2014;52(11):3944-3951.

57. Laird GM, Rosenbloom DIS, Lai J, Siliciano RF, Siliciano JD. Measuring the Frequency of Latent HIV-1 in Resting CD4+ T Cells Using a Limiting Dilution Coculture Assay. In: Methods in Molecular Biology. 2016:239-253 
646 58. Rosenbloom DIS et al. Designing and Interpreting Limiting Dilution Assays: General

647 Principles and Applications to the Latent Reservoir for Human Immunodeficiency Virus-1. Open

648 Forum Infectious Diseases 2015;2(4):ofv123.

649 59. Bates D, Mächler M, Bolker B, Walker S. Fitting Linear Mixed-Effects Models Using Ime4.

650 Journal of Statistical Software 2015;67(1). doi:10.18637/jss.v067.i01

651 60. Hothorn T, Bretz F, Westfall P. Simultaneous Inference in General Parametric Models.

652 Biometrical Journal 2008;50(3):346-363. 
a.

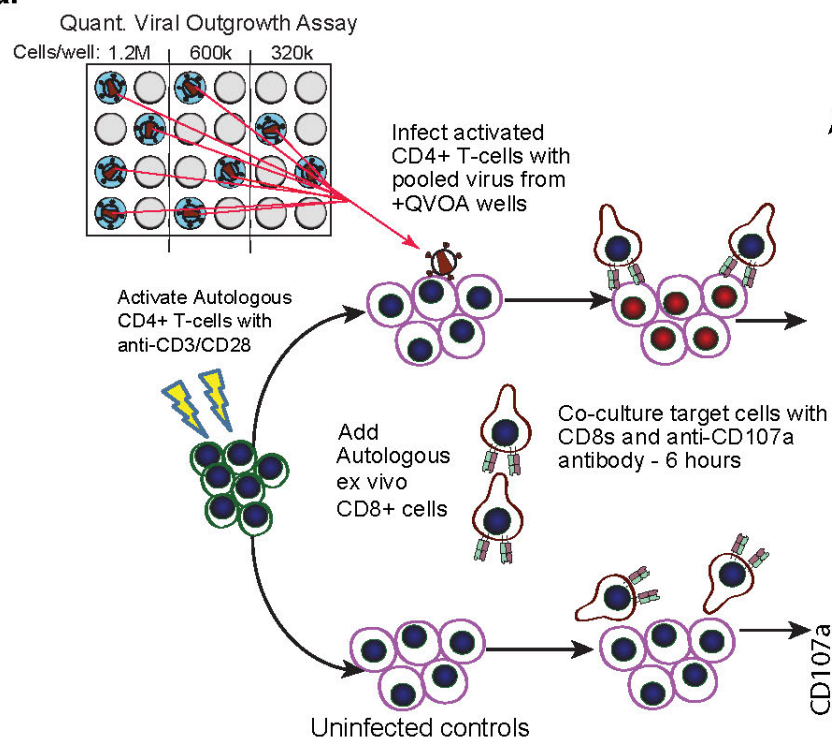

b.
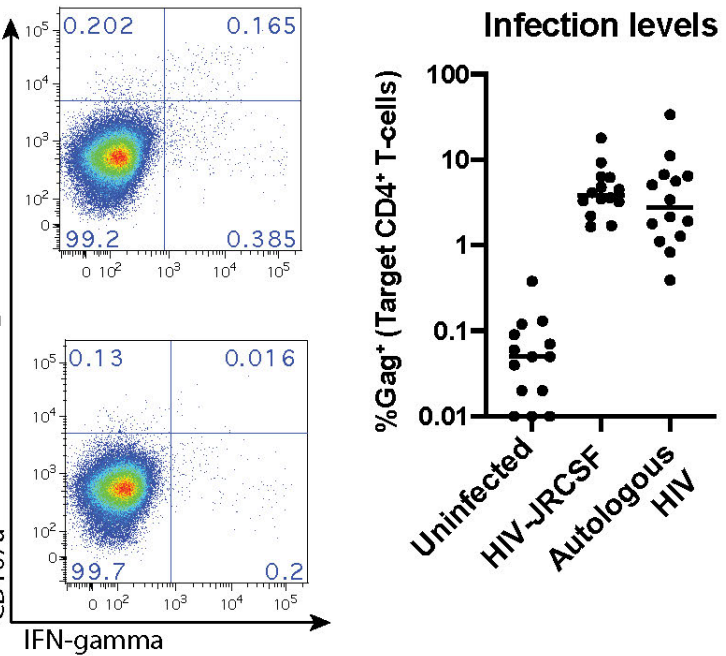

c.

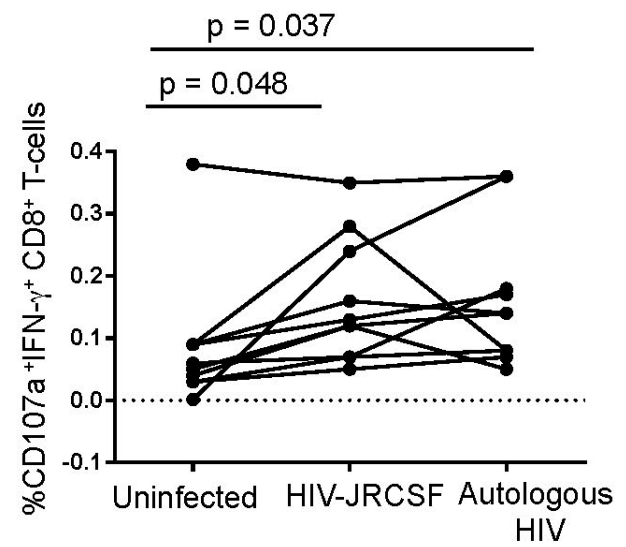

d.

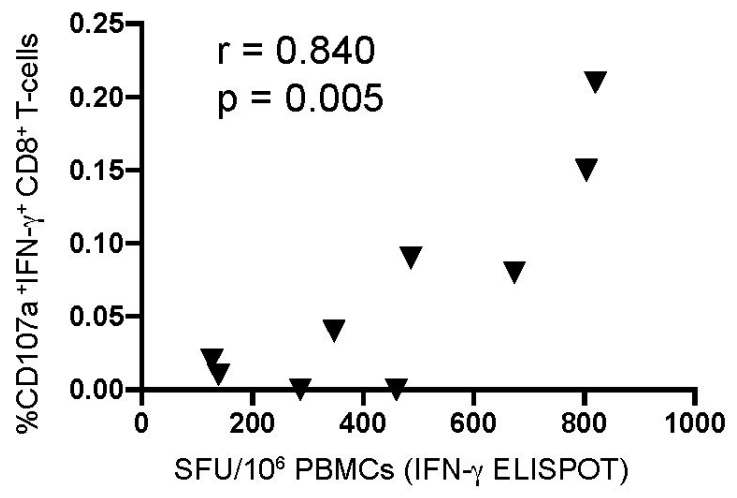

Fig. 1. CD8 ${ }^{+} \mathrm{T}$-cell responses to virus-infected cells can be detected ex vivo, correlating with ELISPOT responses. A. Schematic of 'biosensor assay'. Top-right: Flow cytometry plot gated on $\mathrm{CD}^{+} \mathrm{T}$-cells co-cultured with infected $\mathrm{CD} 4^{+} \mathrm{T}$-cells. Bottom-right: Uninfected control. B. $\mathrm{CD}^{+}$target cells' infection levels from all assays, measured by flow cytometry. C. Flow cytometry data depicting $\% \mathrm{CD} 107 \mathrm{a}^{+} \mathrm{IFN}-\gamma^{+}$of viable $\mathrm{CD} 8^{+} \mathrm{T}$-cells, each line representing a different

681 participant (means from 3 replicates). P-values calculated by RM one-way ANOVA with Dunnett's 
682 multiple comparison test. D. Spearman's correlation between background (uninfected) subtracted

683 responses to autologous HIV (as in C), and summed IFN- $\gamma$ ELISPOT responses to HIV proteome.

684

685

686

687

688

689

690

691

692

693

694

695

696

697

698

699

700

701

702

703

704

705

706

707 


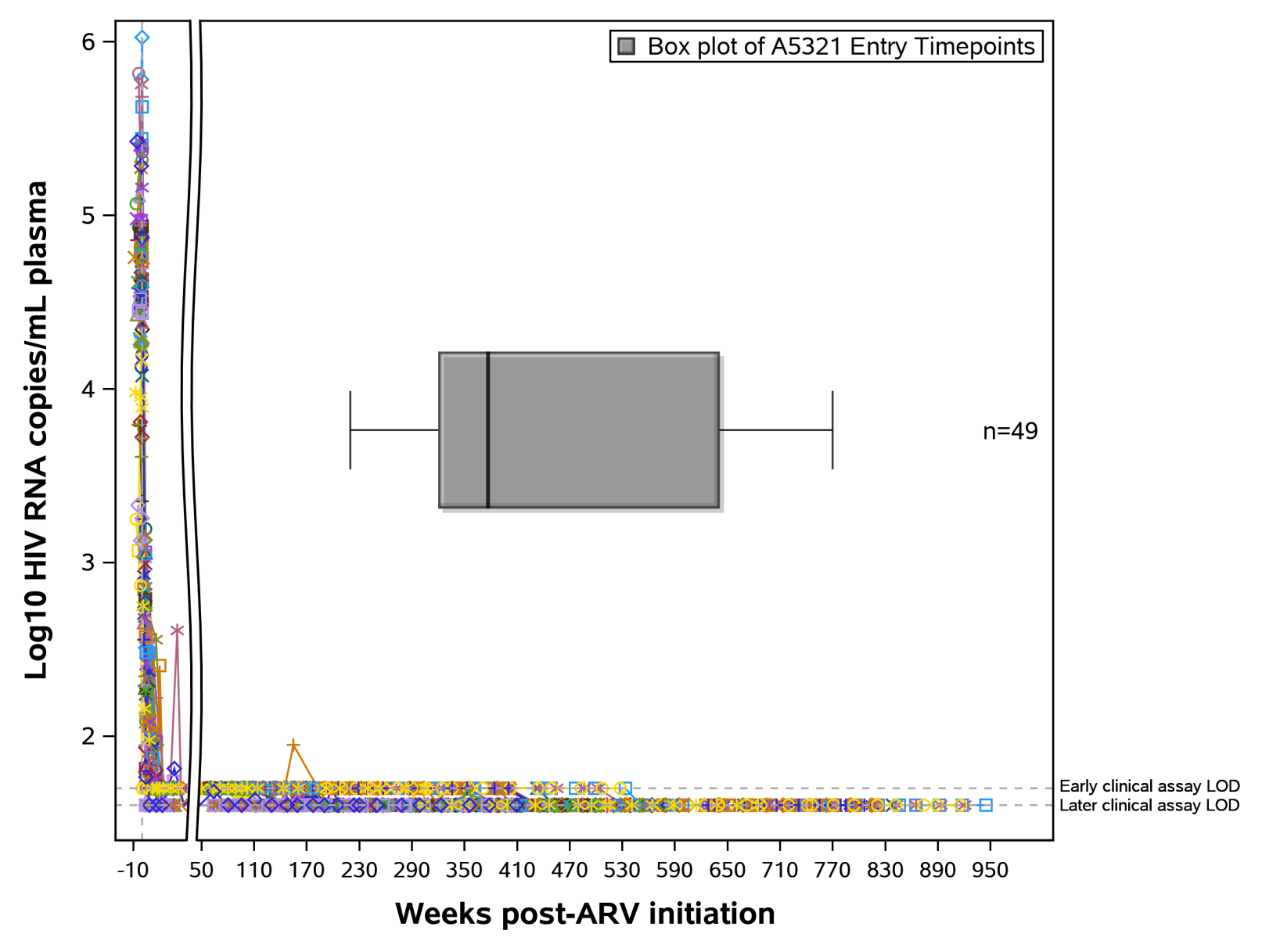

709 Fig. 2. ACTG A5321 Cohort participants achieved viral suppression prior to study entry

710 and maintained viral suppression throughout the study period. $\log _{10}$ plasma HIV RNA

711 (copies $/ \mathrm{mL}$ ) by clinical commercial assays for ACTG A5321 Cohort study participants included

712 in this longitudinal sub-study, followed from pre-ART initiation (ART initiated in other ACTG

713 trials) through to the A5321 study 168 week timepoint. Limit of detection (LOD) for early clinical

714 assays was 50 copies $/ \mathrm{mL}$, and for later clinical assays 40 copies $/ \mathrm{mL}$. Colored lines represent

715 individual participants $(\mathrm{n}=49)$, with symbols indicating each clinical viral load measurement. X-

716 axis break shows time post-ART initiation when all participants achieved initial viral suppression.

717 Box plot shows the distribution of participants' A5321 study entry timepoints relative to weeks

718 post-ART initiation (minimum, Q1, median, Q3, maximum). 

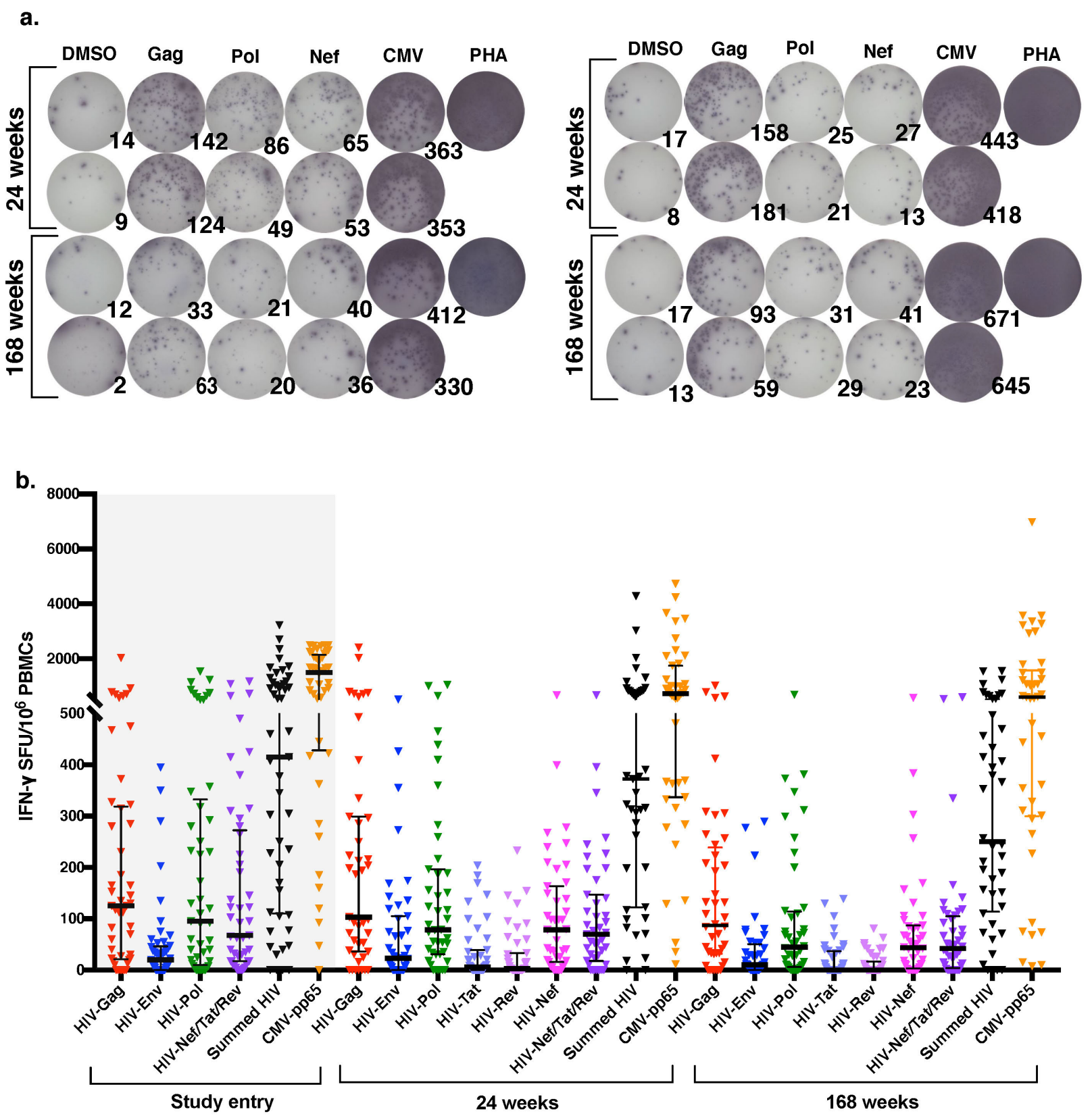

720 Fig. 3. HIV-specific T-cell responses readily detectable ex vivo and persist on long-term

721 ART, primarily directed against HIV-Gag, HIV-Pol, and HIV-Nef. A. Representative IFN- $\gamma$

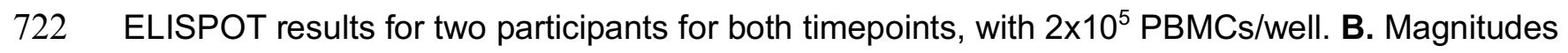

723 of IFN- $\gamma$ responses are shown for three on-ART timepoints. Study entry timepoint data is shaded 
724 in gray because it was not performed in batch with 24 and 168 weeks timepoints. Each data point

725 represents the mean spot forming units (SFU)/10 6 PBMCs following background subtraction of

726 negative control wells (duplicates). Vertical lines and error bars represent median and interquartile

727 range for each peptide pool. 

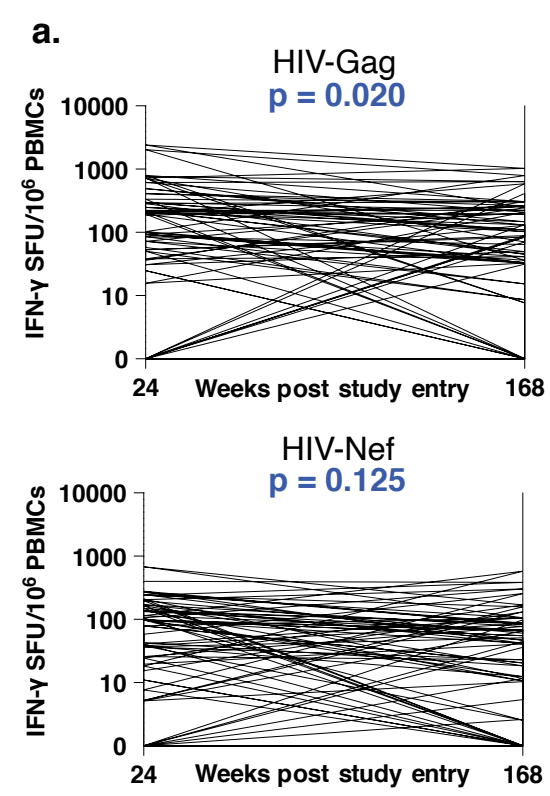

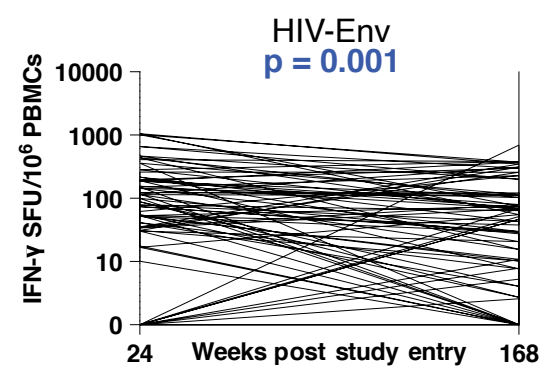

Summed HIV

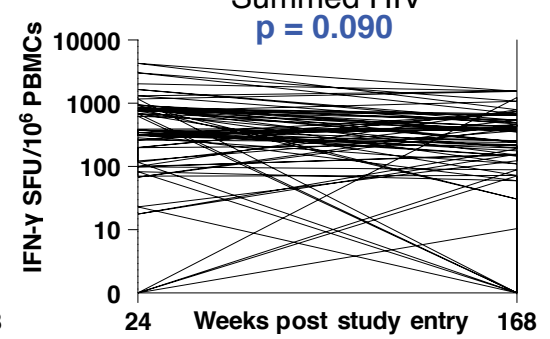

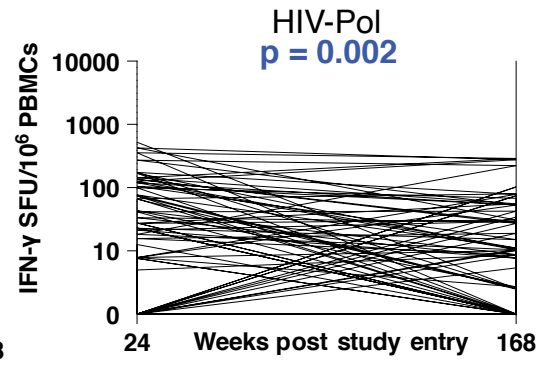

CMV-pp65

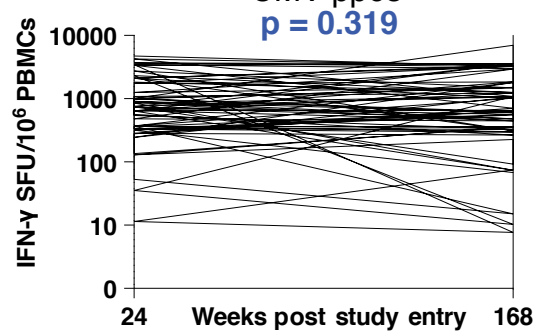

b.

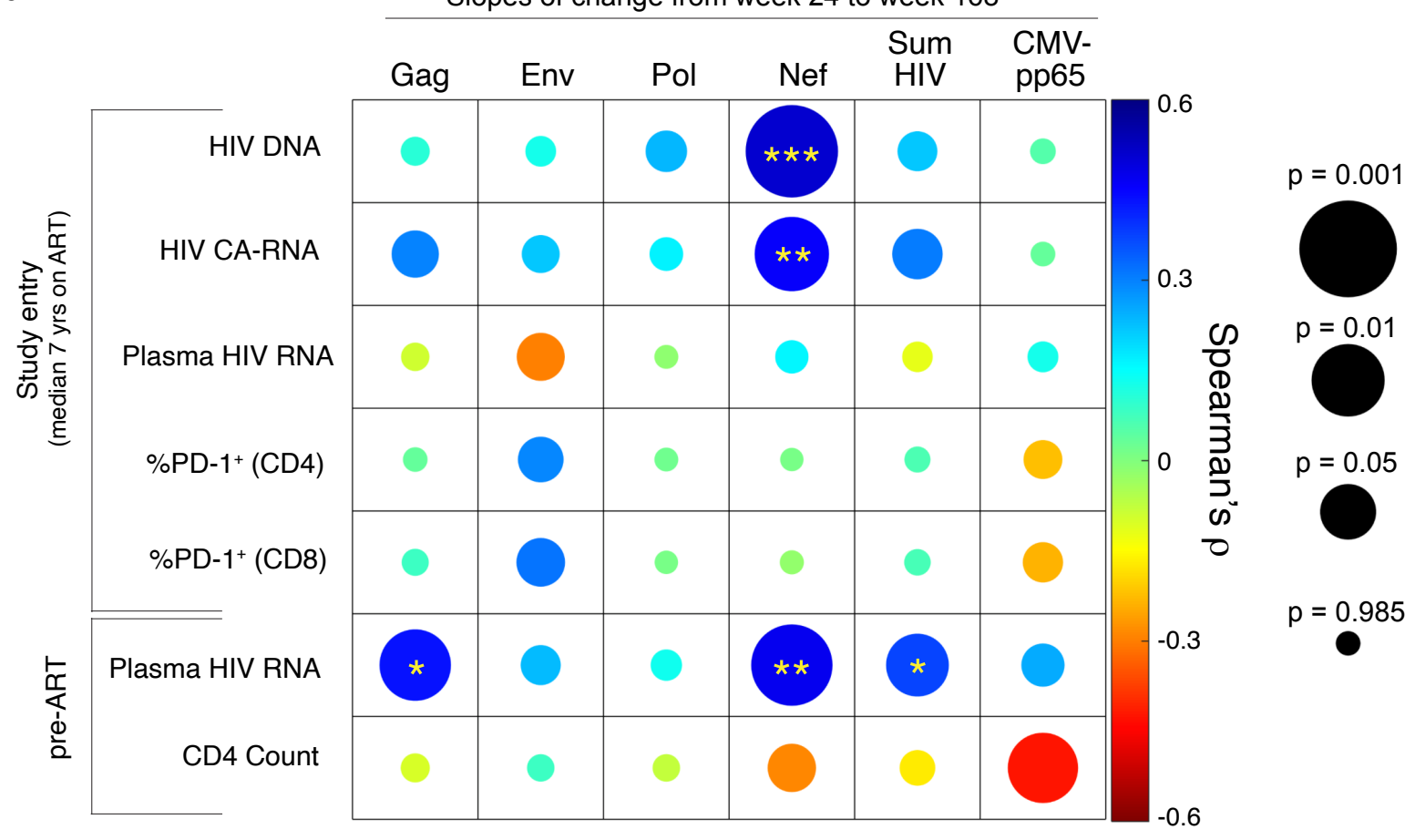

751 Fig. 4. HIV-specific T-cell responses highly stable on long-term ART, with HIV-Nef-specific

752 response dynamics uniquely associated with reservoir measures. A. Participant-specific

753 slopes of change in T-cell responses from weeks 24 to 168 post-study entry. P-values represent 
754 the significance level for the covariate time (in weeks) in linear mixed-effects models from Table

755 S4. B. Correlogram depicting Spearman correlations between slopes of change in raw

756 magnitudes of T-cell responses (from panel A) with virologic and immunologic parameters. Color

757 scale bar represents magnitude of correlation coefficient. Circle size represents unadjusted p-

758 values. Asterisks represent adjusted p-values controlling for pre-ART plasma HIV RNA and CD4 ${ }^{+}$

759 T-cell count $\left({ }^{*}<0.05,{ }^{* *}<0.01,{ }^{* * *}<0.001\right)$.

760

761

762

763

764

765

766

767

768

769

770

771

772

773

774

775

776

777

778

779 


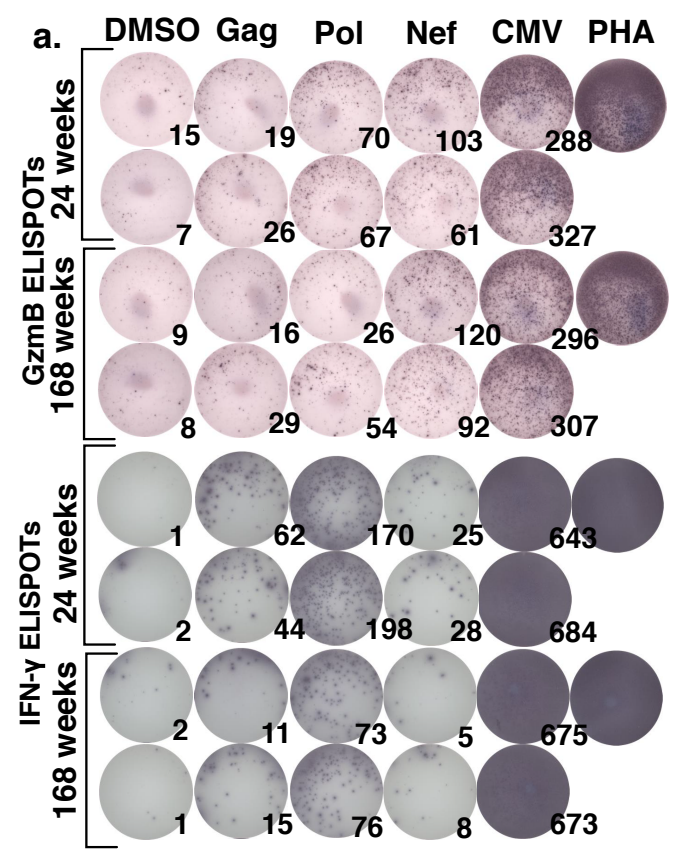

c.
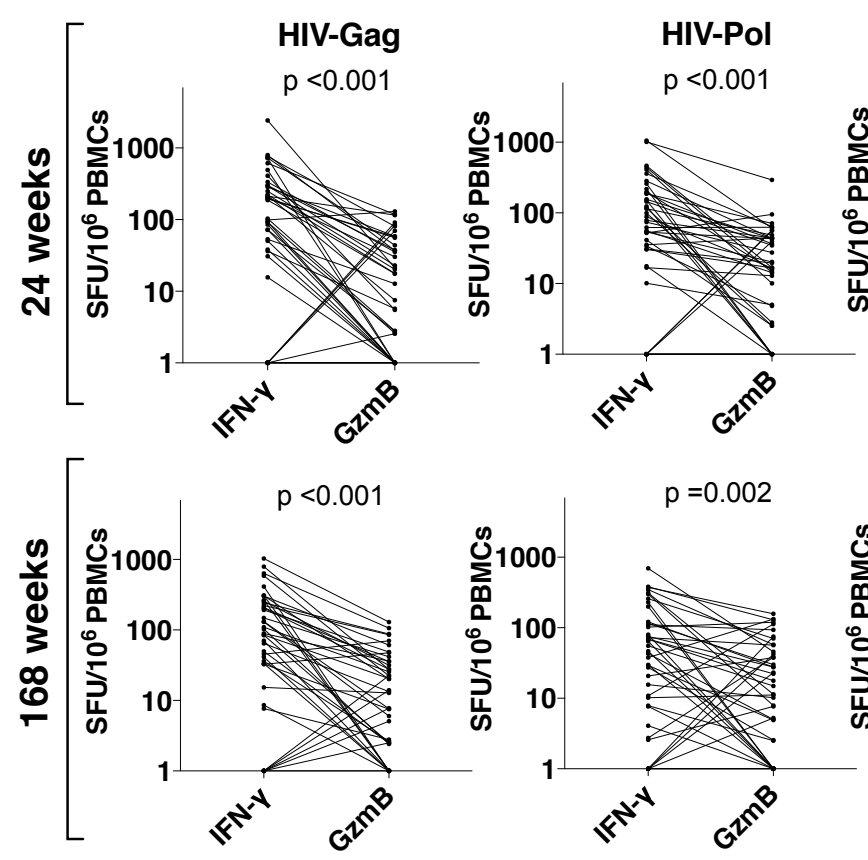

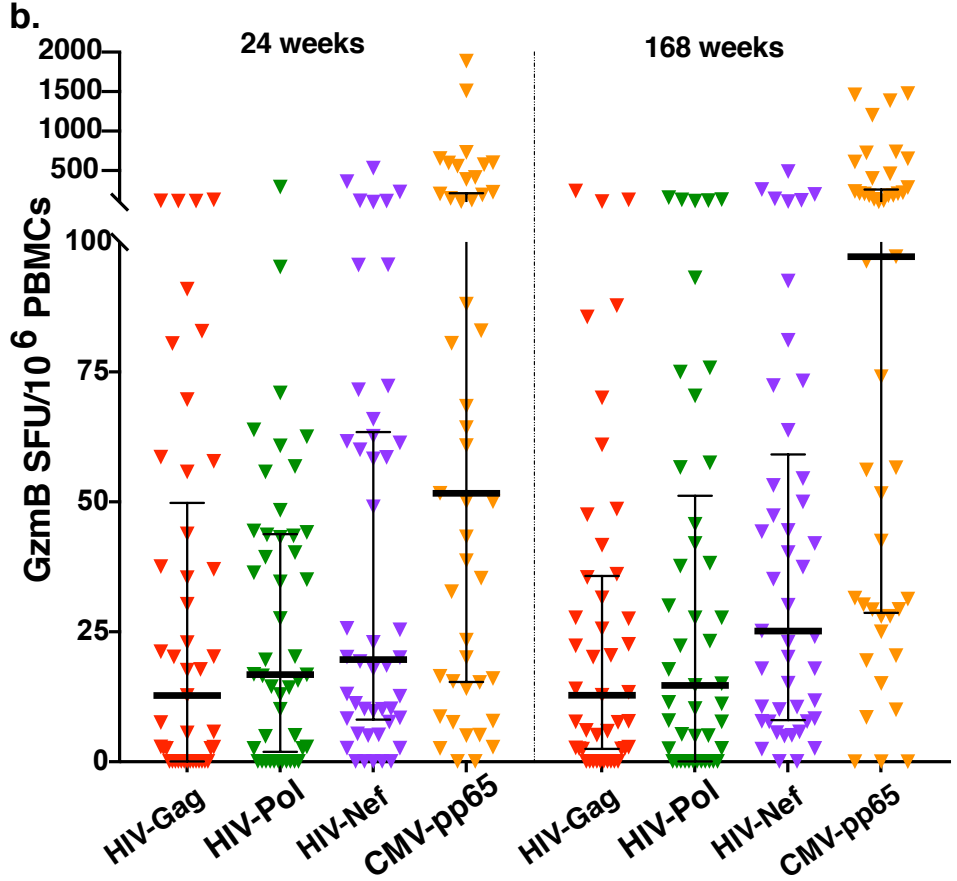

HIV-Nef
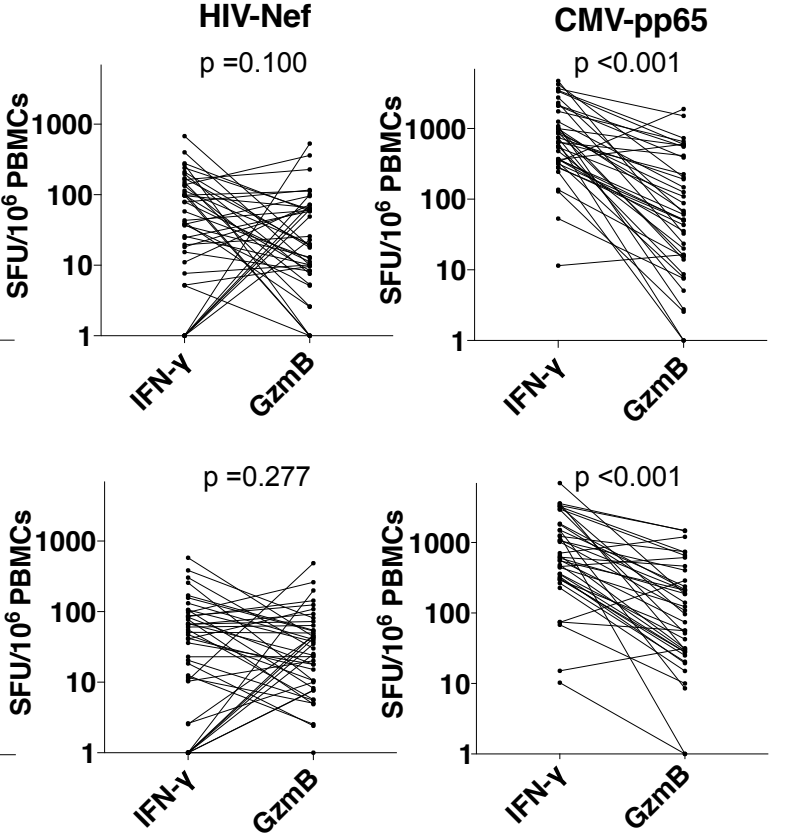

781 Fig. 5. HIV-specific T-cells demonstrate cytotoxic ability, preferentially directed towards

782 HIV-Nef, evidencing recent in vivo antigen exposure. A. Corresponding granzyme B and IFN-

$783 \gamma$ ELISPOT results for one participant at both timepoints, with $2 \times 10^{5} \mathrm{PBMCs} /$ well. B. Magnitudes

784 of granzyme B responses are shown for two batched on-ART timepoints. Each data point

785 represents the mean number of SFU $/ 10^{6}$ PBMCs following background subtraction of mean of 
786 negative control wells. Vertical lines and error bars represent median and interquartile range for

787 each peptide pool. C. Pairwise comparisons of granzyme B versus IFN- $\gamma$ responses for Gag, Pol,

788 Nef, and CMVpp65 at both timepoints. P-values calculated by Wilcoxon matched pairs signed-

789 rank test.

790

791

792

793

794

795

796

797

798

799

800

801

802

803

804

805

806

807

808

809

810 
bioRxiv preprint doi: https://doi.org/10.1101/2020.08.28.272625; this version posted August 28, 2020. The copyright holder for this preprint (which was not certified by peer review) is the author/funder. All rights reserved. No reuse allowed without permission.

\section{Tables}

812

\begin{tabular}{|c|c|c|c|c|c|c|c|c|}
\hline \multirow[t]{2}{*}{ Continuous Variables } & \multirow[t]{2}{*}{ Median } & \multicolumn{2}{|c|}{ Range } & \multicolumn{2}{|c|}{ Missing } & \multirow[t]{2}{*}{ Categorical Variables } & \multirow[t]{2}{*}{$\overline{\mathrm{n}}$} & \multirow[t]{2}{*}{$\%$} \\
\hline & & Lower & Upper & $\mathrm{n}$ & $\%$ & & & \\
\hline Age at A5321 entry (years) & 48 & 23 & 74 & 0 & $0.00 \%$ & Sex & & \\
\hline Years on ART at A5321 entry & 6.6 & 4.2 & 14.8 & 0 & $0.00 \%$ & Female & 11 & $22.45 \%$ \\
\hline HIV CA-DNA at A5321 entry (cps/ $10^{6} \mathrm{CD} 4+$ T-cells) & 515.7 & 5.2 & 5494.0 & 0 & $0.00 \%$ & Male & 38 & $77.55 \%$ \\
\hline HIV CA-RNA at A5321 entry (cps/10 $10^{6} \mathrm{CD} 4+\mathrm{T}$-cells) & 24.2 & 13.6 & 898.9 & 2 & $4.08 \%$ & Race/Ethnicity & & \\
\hline HIV plasma RNA via isCA at A5321 entry (cps/mL) & 0.4 & 0.4 & 8.8 & 3 & $6.12 \%$ & American Indian/Alaskan Native & 1 & $2.04 \%$ \\
\hline$\% \mathrm{PD}-1+\mathrm{CD} 4+$ cells at A5321 entry & $36.75 \%$ & $1.20 \%$ & $83.10 \%$ & 5 & $10.20 \%$ & Black (non-Hispanic) & 5 & $10.20 \%$ \\
\hline$\% \mathrm{PD}-1+\mathrm{CD} 8+$ cells at A5321 entry & $35.40 \%$ & $0.70 \%$ & $84.90 \%$ & 5 & $10.20 \%$ & Hispanic (regardless of Race) & 16 & $32.65 \%$ \\
\hline Pre-ART plasma HIV-1 RNA $\left(\log _{10} \mathrm{cps} / \mathrm{mL}\right)$ & 4.6 & 2.3 & 5.9 & 0 & $0.00 \%$ & White (non-Hispanic) & 27 & $55.10 \%$ \\
\hline Pre-ART CD4+ T-cell count (cells $/ \mathrm{mm}^{3}$ ) & 287.5 & 15.5 & 708.5 & 0 & $0.00 \%$ & $\begin{array}{l}\text { iSCA qualifier at A5321 entry } \\
\text { Undetectable }\end{array}$ & 22 & $44.90 \%$ \\
\hline & & & & & & Detectable & 27 & $55.10 \%$ \\
\hline
\end{tabular}

813

814

815

816

817

818

819

820

821

822

823

824

825

826

827

828

829

830

831 